\title{
Control of Airborne Noise Generated in the Cavities of a T-Slotted Aluminum Cylindrical Column
}

\author{
Mohamad Y. Mustafa, Yizhong Xu, Bjørn Reidar \\ Sørensen, Jose Santos \\ Institute of Buildings, Energy and Material Technology \\ UiT The Arctic University of Norway \\ Narvik, Norway \\ mohamad.y.mustafa@uit.no
}

\author{
Aziz Al-Mahadin \\ Aviation Engineering Department, DBM \\ Higher Colleges of Technology \\ Dubai, UAE \\ aalmahadin@hct.ac.ae
}

\begin{abstract}
This paper presents a study of airborne noise generated by a T-slotted cylindrical aluminum hollow column with six slots of the type used for the construction of communication towers. Noise is a very distracting phenomena and an environmental concern caused by various sources. Noise pollution is becoming more severe with the introduction of massive human activities and the use of heavy machineries, and hence, it has been investigated by many researchers to highlight its environmental impacts and to find methods for its attenuation. Worldwide research focus on noise measurement, comparison of the efficacy of different noise barriers, development of noise models, and proposing viable solutions for its attenuation. Many technologies have been utilized for noise mitigation including insulation, absorption, damping and isolation. In this research, to mitigate the airborne noise level, passive noise control methods are applied by changing boundary conditions and using different materials in the bottom of the cavities. The wind tunnel experiments and CFD modelling reveals that such passive noise control measures have significant effects on controlling the noise level. The investigation also includes CFD modeling for the impact of local velocity distribution in the cavity regions on the noise level.
\end{abstract}

Keywords-airborne noise; Computational Fluid Dynamics (CFD); sound control; Modal analysis; Helmholtz resonance; noise attenuation

\section{INTRODUCTION}

Noise is defined as the unwanted sound judged to be unpleasant, loud or disruptive to hearing. Exposure to noise has been identified as a public health issue in modern societies, which has multiple negative effects on human body. Depending on duration and level of exposure, noise may cause or increase the likelihood of hearing loss, high blood pressure, ischemic heart disease, sleep disturbances, injuries, etc. It is also found that noise causes psychological problems such as annoyance, psychiatric disorders, and effects on psychosocial well-being [1].

Environmental noise is a major type of pollution closely affecting human daily life. It is defined as the accumulation of all noise presented in a specified environment. The European Environment Agency regulates noise control and surveillance within the European Union. EU has set the Environmental Noise Directive to determine levels of noise exposure, increase awareness of environmental noise, and reduce environmental noise. There are many mitigation strategies and controls that can be taken to reduce noise levels including: source intensity reduction, implementation of planning strategies, noise barriers and sound baffles, time of day use regimes, vehicle operational controls, and architectural acoustics design measures [1].

Airborne noise is a significant source of environmental pollution created when a source oscillates and changes the pressure of the surrounding air, as in Fig. 1. These changes in pressure are perceived as sound by the human ear when they are within a certain range from $20 \mathrm{~Hz}$ to $20000 \mathrm{~Hz}$ [1]. Noise is most commonly discussed in terms of decibels $(\mathrm{dB})$. One important parameter of sound is the sound pressure level (SPL) which can be measured by decibels. SPL is associated with the distribution of the surrounding media velocity and the perceived loudness, which varies with the frequency in a nonuniform way, making some frequencies more disturbing than others [2]. As such, mitigating airborne noise requires actions on controlling the distribution of air velocity and the induced frequency.

Noise can be generally controlled by using either passive or active control systems. Active control system uses externally activated sources to minimize the noise, whereas passive noise control system relies on materials to reduce or eliminate the noise. The first method is good at controlling the low frequency noise, and the latter works for the mid and high frequency ranges [3].

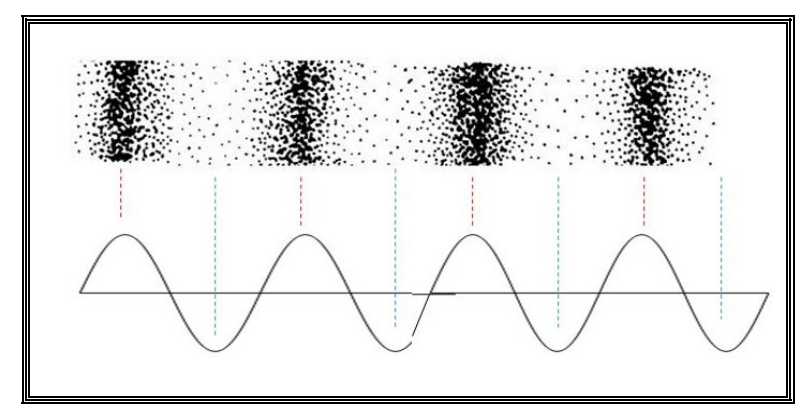

Fig. 1. Change in density of air molecules when affected by a sound source. The molecules shown at the top, whereas the correspondent air pressure variation at the bottom [1]. 
By using both methods, the full spectrum of audible frequencies can be controlled. The most common passive noise control methods include the use of porous media (e.g. porous sheets and resonance absorbers) [4], perforated plates [5] and changes in the geometry of the body (e.g. Helmholtz resonator in mufflers) [6] [7]. In addition to these solutions, the use of the right material can provide additional benefits due to its properties and by using flexible boundaries or flexible walls.

This paper presents an investigation of passive noise control of airborne noise induced by an aluminum cylindrical hollow column with six axial cavities. This type of cylinder is commonly used in the communication industry to construct communication towers. The slots are designed to clamp special clamps and attachments for connecting and supporting the columns. The passive noise control method was achieved by changing the boundary conditions around the six axial cavities in the column as shown in Fig. 2. The boundary conditions were changed by rotating the column at different angles and by modifying the cavities through using different cavity designs, flexible walls and changing the materials in the cavity regions. Noise induced by fluid flow over obstacles is a common engineering problem. Such flow causes unwanted noise in ducts, industrial items, mechanical systems, and in all engineering and life applications. Accordingly, it will be beneficial to model some simple cases to help simulation and investigation of more difficult cases. This investigation was performed using wind tunnel experiments and Computational Fluid Dynamics (CFD) simulations. This paper focuses mainly on the part of CFD simulations.

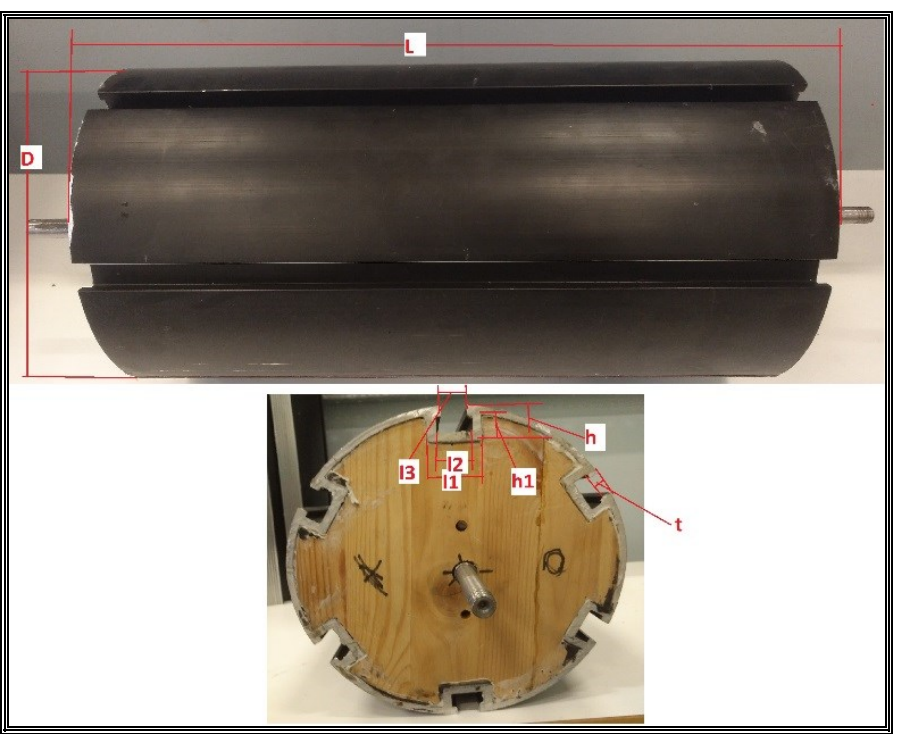

Fig. 2. Configuration of the original aluminum cylindrical hollow column.

\section{EXPERIMENTAL SETUP AND WIND TUNNEL TESTING}

Fig. 2 shows the original aluminum cylindrical hollow column with six axial cavities. The length of the column $\mathrm{L}$ is $450 \mathrm{~mm}$, and the diameter D is $230 \mathrm{~mm}$. Six T- shaped axial cavities are equally distributed in the column, where the widths of the cavity 11,12 and 13 are $35 \mathrm{~mm}, 25 \mathrm{~mm}$ and $20 \mathrm{~mm}$, respectively. The depths of the cavity $h$ and $h 1$ are $25 \mathrm{~mm}$ and $20 \mathrm{~mm}$, and the thickness of the skin is $5 \mathrm{~mm}$. High levels of whistling noise was reported by the users of the aluminum cylindrical elements under investigation. This noise was produced when air impacted the cylinder from different angles.

Passive noise control methods were applied in the investigations by modifying the boundary conditions in the cavity regions as shown in Fig.3a, where sixteen thin ditches with the depth of $5 \mathrm{~mm}$ and width of $5 \mathrm{~mm}$ were arranged in the surface of the column at the two sides of each cavity. Fig. 3b shows polymer sheets covering the bottom of each cavity, which changed the material in the target regions; this changed the thickness of boundary layer in the cavity regions, which resulted in changing the structure of air velocity and essentially affected the airborne noise in these regions.

The investigation was conducted in the open channel wind tunnel at the Arctic University of Norway. During the experiments, the velocity of air in the wind tunnel was monitored by a digitalized pitot static manometer, and the noise was measured by a Bruel \& Kjaer 2260 Sound Analyser, with a spectrum of $\mathrm{dB}$ and frequency. The measurement equipment is shown in Fig. 4.

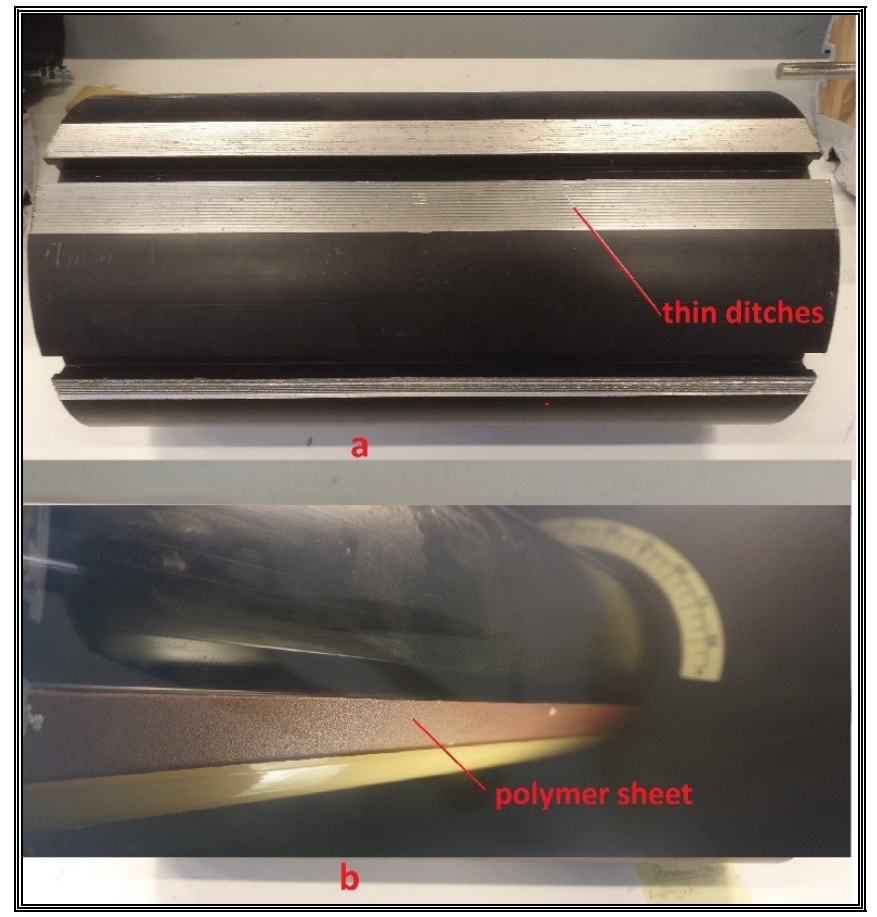

Fig. 3. Passive noise control methods applied to the column. a) Modification of the boundary conditions in the cavity regions, b) Using polymer sheets to cover the bottom of each cavity.

The first step of each testing case was the positioning of the cylinder with respect to airflow direction. When the cavity faced normally to the main direction of airflow, air velocity was increased from $5 \mathrm{~m} / \mathrm{s}$ at steps of $1 \mathrm{~m} / \mathrm{s}$ to measure the spectrum of $\mathrm{dB}$ and the frequency. When the noise level was significantly observable, the velocity was reduced at steps of $0.1 \mathrm{~m} / \mathrm{s}$ and eventually the threshold of air velocity at which unpleasant noise erupted was recorded. For each testing case, the investigation was repeatedly performed in sequence by rotating the column clockwise at a step of 10 degree. 


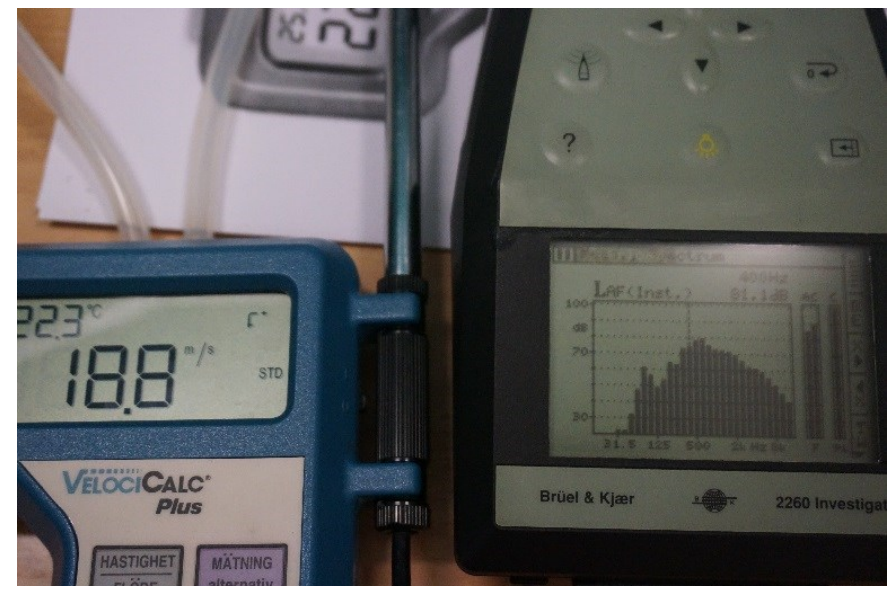

Fig. 4. Noise measurement equipment used in the wind tunnel experiments, Bruel \& Kjaer 2260 Sound Analyser.

Table I shows a summary of minimum threshold velocity where the significant airborne noise was generated for each case. The experiments revealed that the airborne noise level was sensitive to the position of cavities encountered to the main direction of the flow. The proposed passive noise control measures mitigated the noise level at the same velocity of the main flow.

TABLE I. SUMMARY OF THE EXPERIMENTS.

\begin{tabular}{|c|l|c|c|}
\hline No & \multicolumn{1}{|c|}{ Case } & $\begin{array}{c}\text { Threshold } \\
\text { velocity } \mathbf{( m / s )}\end{array}$ & $\begin{array}{c}\text { Rotating } \\
\text { degree } \mathbf{(}^{\circ}\end{array}$ \\
\hline $\mathbf{1}$ & Original case & 15.1 & 80 \\
\hline $\mathbf{2}$ & With thin ditches & 16.3 & 80 \\
\hline $\mathbf{3}$ & $\begin{array}{l}\text { Slot edges covered by } \\
\text { polymer sheet }\end{array}$ & 15.8 & 80 \\
\hline
\end{tabular}

\section{CFD MODELLING AND DISCUSSION}

While physical experiments may yield reliable and realistic data for the subject research, they can have disadvantages such as workforce demand, considerable expenses in preparation and time consuming. Computational Fluid Dynamics is a very important and useful engineering tool that helps in solving fluid flow problems involving geometrically complex cases. In this type of research, CFD tools have been widely accepted as a flexible and inexpensive alternative to wind tunnel testing with great success [2] [3]. The CFD investigation in this paper mainly focused on the impact of local velocity distributions around the slots on the airborne noise, which was not revealed in the wind tunnel experiments.

\section{A. CFD model creation and simulation procedures}

The aluminum cylindrical hollow column was created in a 2D model under the platform of ANSYS fluent software package. The configuration of the cross section is identical to the real column and the size of the numerical domain is similar to the wind tunnel. Fig. 5 shows the created numerical model meshed by triangular elements.

Since the thickness of the boundary layer has profound effects on the local velocity distribution around the cavity regions, appropriate arrangement of elements in such regions was essential to simulate the effects of the boundary condition on the local velocity distribution. This was fulfilled by inflating 10 layers around the surface of the column body, Fig. 5.

Inflation is feature in Ansys Fluent CFD tool, by which the mesh cells or elements of the boundary layer can be stacked in the direction normal to the boundary. In this process, the mesh can be inflated with several layers starting from the boundary layer surface upto the full thickness of the boundary layer.

Mesh sensitivity analysis was performed by comparing the values of velocity magnitudes and turbulence intensity at the compared positions within the domain. Starting with a coarse mesh, solutions were found for finer meshes until the solutions were stable with minor differences, as the mesh gets finer. It was found that a mesh with 0.4 million elements has reached the mesh independence. It was decided that at this point, the mesh was good enough to capture the finest details of the flow.

The conditions of velocity inlet used were the ones from the wind tunnel experiment. At the pressure outlet, the flow field variables were extrapolated from the adjacent interior cells and the normal gradient was ignored. According to the characteristics of the wind tunnel, turbulence intensity in the freestream was $5 \%$. Both of the backflow turbulence intensity and the backflow turbulent viscosity ratio were set at $5 \%$ in the simulations. A no-slip condition was applied for all of the walls in the domain. The solution methods adapted SIMPLE (SemiImplicit Method for Pressure Linked Equations) scheme pressure-velocity coupling, as it is a widely used in numerical procedures to solve the Navier-Stokes equations (NSE) [4] [5].

The convergence criteria were assessed by monitoring the scaled residuals. Additionally, the history of drag coefficient in the longitudinal direction was also monitored.

The solution initialization ran the hybrid method with 10 iterations. The ultimate solution was obtained on the second order upwind discretization.

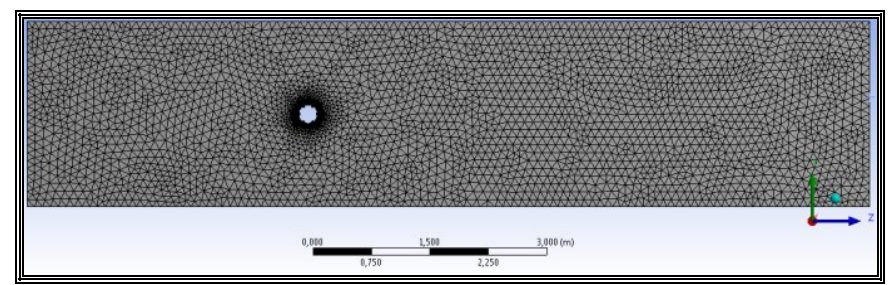

Fig. 4. Overview in 2D of the mesh in the fluid surrounding the cylindrical body.

\section{B. Maximum noise angle}

To find the highest average velocity, the cylindrical body was rotated clockwise in steps of 10 degrees and then the velocities were compared against each other. In the nonmodified body, it was reported that the noise was present at 15 $\mathrm{m} / \mathrm{s}$ but not at $10 \mathrm{~m} / \mathrm{s}$, thus the comparison was done using 15 $\mathrm{m} / \mathrm{s}$ as the initial inlet velocity running from left to right, Fig. 6.

From Fig. 7, the maximum velocity in the cavities occurred at an angle of 80 degrees. It can be noticed that the magnitude 
of the velocity at 70 and 90 degrees was smaller than the ones at 80 degrees. The angle was measured clockwise from the horizontal axis as shown in Fig.6.

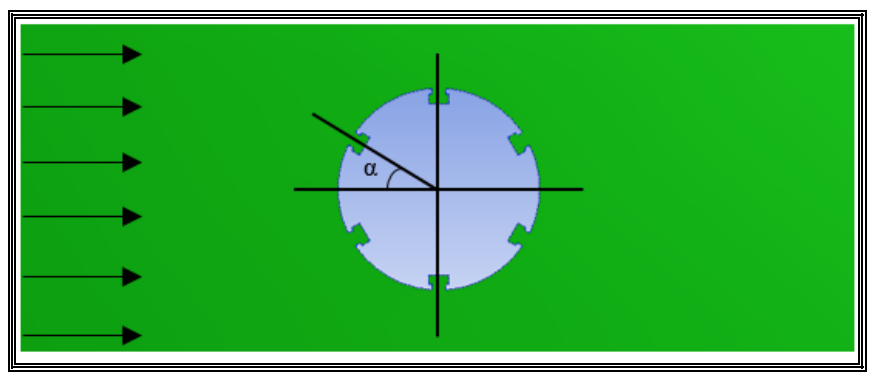

Fig. 5. Sketch showing the direction of the wind and the measured angle for a cavity.

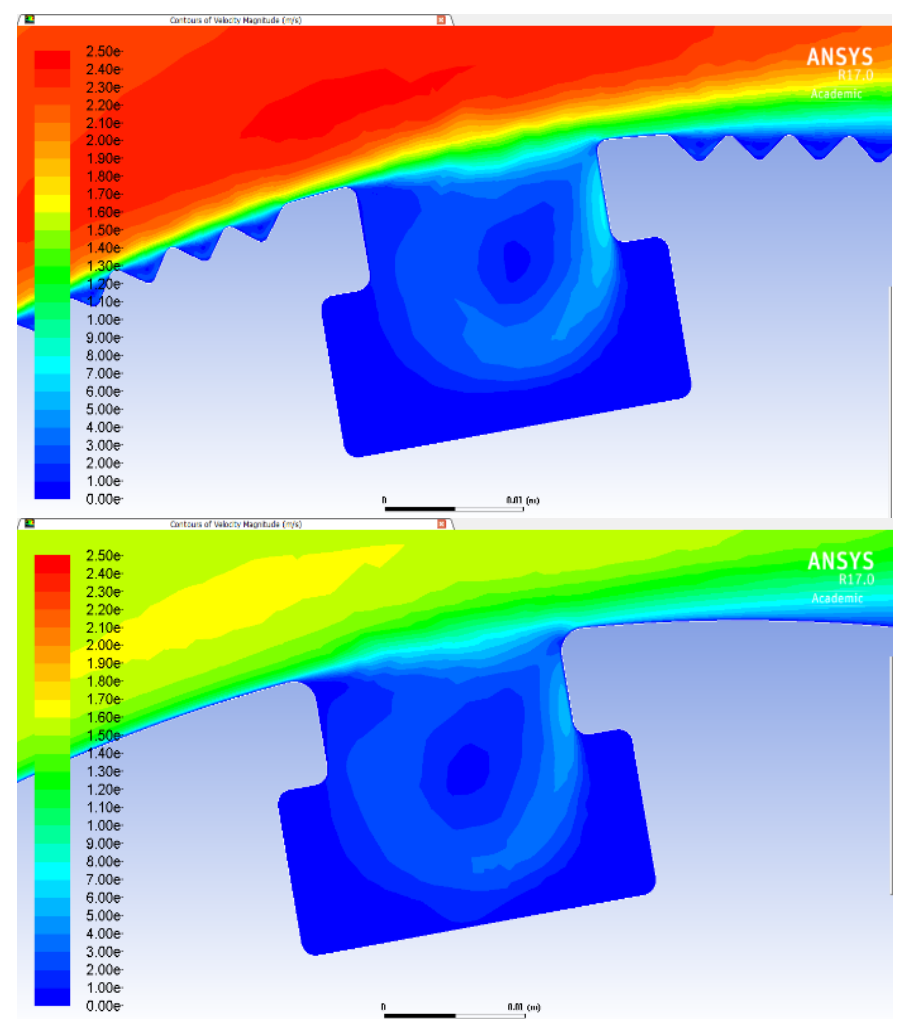

Fig. 6. Velocity contours in the cavities at 70,80 and 90 degrees with an inlet velocity of $15 \mathrm{~m} / \mathrm{s}$ running from left to right.

\section{Noise predominant frequency}

The cavity can be modeled as a Helmholtz Resonator [8] in order to determine the frequency of the section under study. The frequency generated by the wind is obtained by substituting the necessary values in the equation for the Helmhotz resonator as follows [9]:

$$
f=\frac{c}{2 \pi} \sqrt{\frac{A}{V L}}
$$

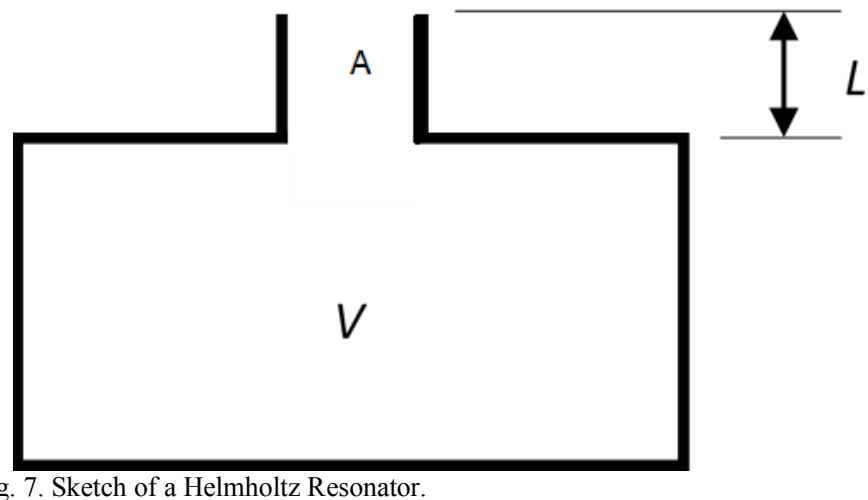

where $\mathrm{A}$ is the cross section area of the neck, $\mathrm{V}$ is the volume of the cavity (excluding the neck), $\mathrm{L}$ is the height of the neck and $\mathrm{c}$ is the speed of sound, as in Fig. 8.

Using a simplified geometry, without considering the small radii in the corners, it was found that the natural frequency for the slots was very close to $630 \mathrm{~Hz}$. The modal analysis for the slotted cylinder can be performed using standard CFD software. After running a modal analysis using ANSYS on the initial geometry, it was possible to determine the natural frequencies of the body. Modal analysis is a technique to study the dynamic characteristics of a structure under vibrational excitation. Using this analysis, natural frequencies, mode shapes and mode vectors of a structure can be determined. Modal analysis allows the design to avoid resonant vibrations or to vibrate at a specified frequency and gives engineers an idea of how the design will respond to different types of dynamic loads [10].

The frequencies of the first ten mode shapes obtained using the modal analysis feature in ANSYS are presented in Table II. It can be noticed that the calculated natural frequency of the cylindrical body (i.e. $630 \mathrm{~Hz}$ ) is enclosed by the frequencies of the 5th and 6th modes. Both of these modes have a difference of $3 \%$ from the theoretical value, thus the body will oscillate

TABLE II. TEN MODES AND FREQUENCIES OF THE ORIGINAL CYLINDRICAL COLUMN OBTAINED USING MODAL ANALYSIS IN ANSYS.

\begin{tabular}{|c|c|}
\hline Mode & Frequency $[\mathbf{H z}]$ \\
\hline $\mathbf{1}$ & 370.01 \\
\hline $\mathbf{2}$ & 370.09 \\
\hline $\mathbf{3}$ & 583.84 \\
\hline $\mathbf{4}$ & 613.23 \\
\hline $\mathbf{5}$ & 613.26 \\
\hline $\mathbf{6}$ & 646.71 \\
\hline $\mathbf{7}$ & 942.95 \\
\hline $\mathbf{8}$ & 985.12 \\
\hline $\mathbf{9}$ & 985.33 \\
\hline $\mathbf{1 0}$ & 1068.9 \\
\hline
\end{tabular}

\section{Proposed solution}

The suggested solution to eliminate the noise was by carving a pattern on the external surface of the cylinder to reduce air velocity in the neck of the cavity and hence shift the natural frequency to a new range which can be outside the range attained within prevailing wind conditions. A total of 72 
triangular axial ditches were machined parallel to each of the cavities in the exterior of the cylinder and were equally distributed in groups of 6 on each side of the cavities. The ditches caused the air layer to flow at a lower velocity in the neck of the cavity. This low velocity was not sufficient to force the air in the neck to oscillate and create noise, hence noise creation was inhibited. Fig. 9 is a frontal cross section of the modified geometry that shows the axial ditches, six on each side of the cavity.

By comparing the magnitudes of the velocity contours in the neck of the cavity in Fig. 10 (modified body, $15 \mathrm{~m} / \mathrm{s}, 80$ degrees) with the ones in Fig. 11 (non-modified body, $10 \mathrm{~m} / \mathrm{s}$, 80 degrees), it can be observed that the velocity contours are similar for both cases. Which indicated that the surface modification has eliminated the noise creation conditions at 15 $\mathrm{m} / \mathrm{s}$.

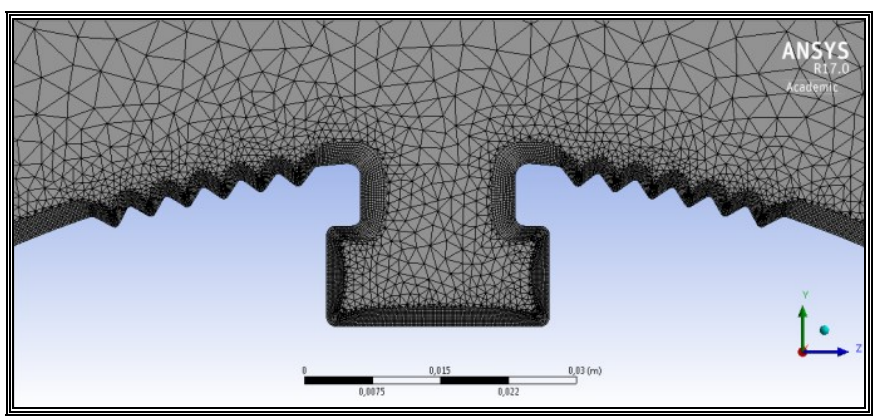

Fig. 8. Frontal cut of the final geometry showing six grooves on each side of the cavity.

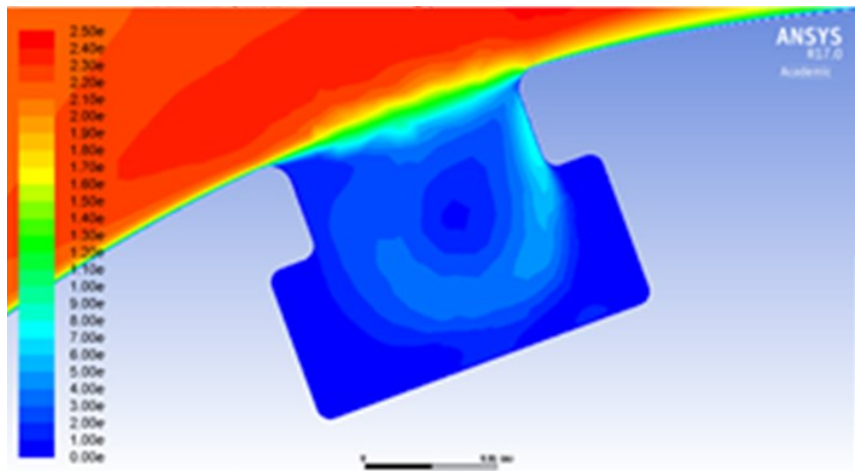

Fig. 9. Velocity contours in the cavity with an inlet velocity of $10 \mathrm{~m} / \mathrm{s}$ and an inclination of 80 degrees.

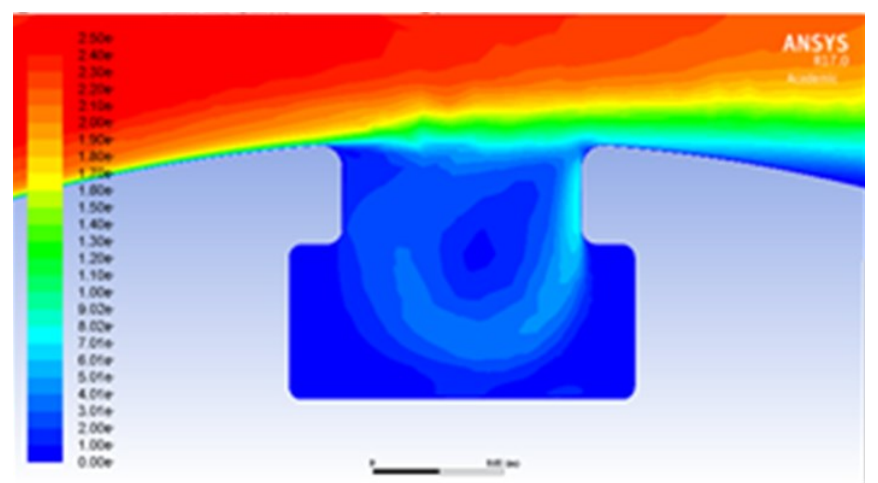

Fig. 10. Velocity contours in the cavity with an inlet velocity of and an inclination of 80 degrees.

\section{SUMMARY AND RECOMMENDATIONS}

This paper has presented an investigation of airborne noise generated by a T-slotted aluminum cylindrical hollow column. This type of column is used in the communication industry to construct communication towers and antennas. It was reported by users that the column elements tend to produce undesirable whistling noise at certain wind conditions. A full analytical, experimental and computational analysis of the structural element were performed to analyze the conditions that lead to noise generation. The natural frequency of the slots was determined using analytical techniques based on Helmholts Resonator phenomenon and using the modal analysis features of ANSYS CFD. To mitigate the generated noise, passive noise control methods were used by changing the boundary conditions adjacent to the slots in order to change the characteristics of wind flow inside the slots, and by changing the material properties, using different materials, in the bottom of the cavities. The wind tunnel experiments and CFD modelling reveal that the proposed measures have significant effects on mitigating the noise level. The proposed method to reduce the noise was achieved by creating a pattern on the external surface of the investigated column along the slots. This pattern reduced the air layer flow velocity at the neck of the cavity. This low velocity was inadequate to force the air in the neck to oscillate and create noise.

The investigation presented in this work covered only a limited setup which was focused on a particular problem, however, the authors realize that many other situations can occur, in which passive noise mitigation is essential. Various set ups can be investigated to establish a better picture of the mitigation methods for airborne noise. Therefore, the following future works are recommended:

- Experimenting the same setup but with moving column.

- Investigating other geometrically complex problems.

\section{CONCLUSIONS}

Both wind tunnel experiments and CFD modelling were investigated. It was found that the airborne noise level is sensitive to the position of the cavities created through the column with respect to the main direction of airflow. The applied passive noise control measures which involved modification of boundary layer conditions and alteration of resonant frequency using damping materials introduced significant effects on mitigating the airborne noise level for the studied column.

\section{ACKNOWLEDGMENT}

The authors would like to express their gratitude to UiT the arctic university of Norway particularly the Institute of Buildings, Energy and Material Technology and the ColdTech project, funded by the Research Council of Norway for supporting this work. 


\section{REFERRENCES}

[1] Passchier-Vermeer, W.; Passchier, W.F.; «Noise exposure and public health,» Environmental Health Perspective, vol. 108, pp. 123-131, 2000.

[2] Blocken, B., «50 years of computational wind engineering: past, present and future,» Wind Engineering and Industrial Aerodynamics, pp. 69-102, 2014.

[3] Bourdin, P.; Wilson, J.; «Windbreak aerodynamcis: is computational fluid dynamics reliable?,» Boundary-Layer Meteorology , pp. 181-208, 2008.

[4] Giannoulis, A.; Mistriotis, A.; Briassoulis, D.; «Experimental and numerical investigation of the airflow around a raised permeable panel,» Wind Engineering and Industrial Aerodynamics, pp. 808-817, 2010.

[5] Gupta, Satish K.; Ray, Sudipta; Chatterjee, Dipankar, «Influence of aiding buoyancy on the suppression of flow separation for power-law fluids around a circular object,» Heat Transfer Engineering, vol. 37, nr. 15, pp. 1267-1279, 2016.

[6] Hansen, C.H. and Goelzer, B.I.F. , «Chapter 10. Engineering noise control,» i Occupational Exposure to Noise: Evaluation, Prevention and Control. , World Health Organisation Special Report, Published by Federal Institute of Occupational Safety and Health, Germany. ISBN 3089701-721-0, 2011.

[7] K. Dasarathy, «Noise Pollution - Causes, Mitigation and Control Measures for Attenuation,» PhD Thesis, 2015.

[8] Ganghua Yu, Deyu Li and Li Cheng, «Internal Resistance Optimization Of A Helmholtz Resonator In Noise Control Of Small Enclosures,» i 14th international congress on sound and vibration ICSV14, Australia, 2007.

[9] M. Monteiro, «Measuring the acoustic response of Helmholtz resonators,» The Physics Teacher 53, 247 (2015); vol. 53, p. 247, 2015.

[10] «Modal Analysis and Harmonic Response Analysis of a crankshaft,» International Journal of Emerging Technology and Advanced Engineering, vol. 5, nr. 6, p. 1, 2015. 